\title{
Sample size calculation for treatment effects in randomized trials with fixed cluster sizes and heterogeneous intraclass correlations and variances
}

Citation for published version (APA):

Candel, M. J. J. M., \& van Breukelen, G. (2015). Sample size calculation for treatment effects in randomized trials with fixed cluster sizes and heterogeneous intraclass correlations and variances. Statistical Methods in Medical Research, 24(5), 557-573. https://doi.org/10.1177/0962280214563100

Document status and date:

Published: 01/01/2015

DOI:

$10.1177 / 0962280214563100$

Document Version:

Publisher's PDF, also known as Version of record

\section{Document license:}

Taverne

Please check the document version of this publication:

- A submitted manuscript is the version of the article upon submission and before peer-review. There can be important differences between the submitted version and the official published version of record.

People interested in the research are advised to contact the author for the final version of the publication, or visit the DOI to the publisher's website.

- The final author version and the galley proof are versions of the publication after peer review.

- The final published version features the final layout of the paper including the volume, issue and page numbers.

Link to publication

\footnotetext{
General rights rights.

- You may freely distribute the URL identifying the publication in the public portal. please follow below link for the End User Agreement:

www.umlib.nl/taverne-license

Take down policy

If you believe that this document breaches copyright please contact us at:

repository@maastrichtuniversity.nl

providing details and we will investigate your claim.
}

Copyright and moral rights for the publications made accessible in the public portal are retained by the authors and/or other copyright owners and it is a condition of accessing publications that users recognise and abide by the legal requirements associated with these

- Users may download and print one copy of any publication from the public portal for the purpose of private study or research.

- You may not further distribute the material or use it for any profit-making activity or commercial gain

If the publication is distributed under the terms of Article $25 \mathrm{fa}$ of the Dutch Copyright Act, indicated by the "Taverne" license above, 


\author{
Math JJM Candel' and Gerard JP van Breukelen'
}

\begin{abstract}
When comparing two different kinds of group therapy or two individual treatments where patients within each arm are nested within care providers, clustering of observations may occur in both arms. The arms may differ in terms of (a) the intraclass correlation, (b) the outcome variance, (c) the cluster size, and (d) the number of clusters, and there may be some ideal group size or ideal caseload in case of care providers, fixing the cluster size. For this case, optimal cluster numbers are derived for a linear mixed model analysis of the treatment effect under cost constraints as well as under power constraints. To account for uncertain prior knowledge on relevant model parameters, also maximin sample sizes are given. Formulas for sample size calculation are derived, based on the standard normal as the asymptotic distribution of the test statistic. For small sample sizes, an extensive numerical evaluation shows that in a two-tailed test employing restricted maximum likelihood estimation, a safe correction for both $80 \%$ and $90 \%$ power, is to add three clusters to each arm for a $5 \%$ type I error rate and four clusters to each arm for a $1 \%$ type I error rate.
\end{abstract}

\title{
Keywords
}

Individually randomized group treatment, maximin design, optimal design, sample size, therapist effects

\section{Introduction}

Many study designs evaluating the effect of an intervention are characterized by observations being correlated within clusters. This may arise in group or cluster randomized trials, ${ }^{1}$ where groups are the units of assignment. In such trials, groups are assigned to one of several treatment conditions and all sampled members of the same group are given the same treatment. For example, school

\footnotetext{
'Department of Methodology and Statistics, School for Public Health and Primary Care CAPHRI, Maastricht University, Maastricht, The Netherlands

Corresponding author:

Math JJM Candel, Department of Methodology and Statistics, Maastricht University, P.O. Box 616, 6200 MD Maastricht, The Netherlands.

Email: Math.Candel@maastrichtuniversity.nl
} 
classes with all of its pupils are assigned to a smoking prevention program, or general practices with all of its sampled patients are assigned to one of several medication regimens. However, when individuals, instead of groups, are the units of assignment, clustering of observations may also occur. This is the case when the treatment itself induces clustering, such as when treatments are given to groups of individuals., ${ }^{2,3}$ In such individually randomized group treatment trials, interactions between persons within a group may lead to the observations on the outcome variable being correlated. ${ }^{4}$ The clustering may occur in only one of the treatment arms, such as when group therapy is compared to a treatment condition involving only medication, ${ }^{5,6}$ but also in both treatment arms, when, for instance, two different types of group therapy are compared..$^{7,8}$ Clustering effects due to treatment may also occur if the treatment is not given to a group as a whole but on an individual basis. If several patients are treated by the same therapist or, more generally, the same care provider, then patients of one therapist will be treated in a more similar way than patients treated by different therapists. Such therapist effects may also lead to correlated observations within clusters. ${ }^{3,4,9}$

The present paper will address sample size calculation for trials comparing group administered interventions or trials where clustering effects are due to therapists, assuming a quantitative outcome variable and estimation of the treatment effect by maximum likelihood (ML) in a linear mixed model analysis. For the case of homogeneity between the treatment arms with respect to outcome variance, intraclass correlation, cluster size, and number of clusters, formulas for sample size calculation have been derived by Raudenbush ${ }^{1}$, Moerbeek et al., ${ }^{10}$ and Liu. ${ }^{11}$ The present study generalizes these results by considering treatment arms that differ in outcome variance, intraclass correlation, cluster size, and number of clusters, for a scenario where the cluster sizes are more or less fixed. This would apply when therapy groups have some ideal group size that is rather fixed, or when therapists have some ideal caseload.

The paper is structured as follows. Section 2 presents the linear mixed effects model for trials comparing two arms, allowing for a difference between both arms with respect to the intraclass correlation, the outcome variance, the number of clusters, and the cluster size. Section 3 gives an explicit expression for the asymptotic variance of the treatment effect estimator and presents the optimal number of clusters for each treatment arm, optimal meaning that these numbers minimize the variance of the treatment effect estimator and thus maximize the test power under cost constraints. Section 4 gives formulas to calculate the optimal number of clusters needed for a given effect size $(E S)$ and power level (as opposed to a given budget) and presents some analytical results for these optimal sample sizes. To handle uncertainty about those model parameters on which the optimal sample size depends, section 5 presents the maximin design as a robust alternative to the optimal design. Section 6 is a numerical evaluation of corrections in sample size calculation for finite samples when starting from the normal distribution as the asymptotic distribution of the test statistic. Section 7 illustrates how to apply the results in sample size calculation and section 8 summarizes the present study's implications for planning trials.

\section{Specification of the linear mixed effects model}

In the treatment and control condition, we have respectively $K_{t}$ and $K_{c}$ clusters (such as $K_{t}$ and $K_{c}$ therapy groups). In each cluster $j\left(j=1, \ldots, K_{t}\right)$ of the treatment condition, there are $m$ persons and in each cluster $j\left(j=K_{t}+1, \ldots, K_{t}+K_{c}\right)$ of the control condition, there are $n$ persons, the total number of persons thus amounting to $N=K_{t} m+K_{c} n$. The dependent variable is a quantitative outcome, denoted as $y_{i j}$ for person $i$ in cluster $j\left(j=1, \ldots, K_{t}+K_{c}\right)$. If in each condition, $y_{i j}$ is 
(approximately) normally distributed, the following linear mixed effects model is an adequate tool for data analysis 3,9

$$
y_{i j}=\beta_{0}+\left(\beta_{1}+u_{j}+\delta_{i j}\right) \operatorname{Int}_{i j}+\left(v_{j}+\varepsilon_{i j}\right)\left(1-\operatorname{Int}_{i j}\right)
$$

where $I n t_{i j}$ denotes the treatment condition for person $i$ in cluster $j$, which is coded as 1 for persons in the treatment condition and coded 0 for persons in the control condition. With this coding scheme, $\beta_{0}$ represents the mean outcome of the control condition and $\beta_{1}$ represents the treatment effect, such as the mean outcome difference between cognitive behavioral therapy and psychodynamic interpersonal psychotherapy. ${ }^{8}$ Commonly, $\beta_{1}$ is the parameter of primary interest. The terms $u_{j}$ and $v_{j}$ represent the random cluster effect in the treatment and control condition, respectively, whereas $\delta_{i j}$ and $\varepsilon_{i j}$ represent a random person effect in these conditions. The effects $u_{j}, v_{j}, \delta_{i j}$, and $\varepsilon_{i j}$ are assumed to be independently normally distributed with respective variances $\sigma_{u}^{2}, \sigma_{v}^{2}, \sigma_{\delta}^{2}$, and $\sigma_{\varepsilon}^{2}$. The total outcome variances for the treatment and control arm are thus $\sigma_{y_{t}}^{2}=\sigma_{u}^{2}+\sigma_{\delta}^{2}$ and $\sigma_{y_{c}}^{2}=\sigma_{v}^{2}+\sigma_{\varepsilon}^{2}$.

In what follows the intraclass correlation, which measures the dependency among observations for members within the same cluster, is relevant. For the model in equation (1), the intraclass correlations are $\rho_{t}=\sigma_{u}^{2} /\left(\sigma_{u}^{2}+\sigma_{\delta}^{2}\right)=\sigma_{u}^{2} / \sigma_{y_{t}}^{2}$ for the treatment arm and $\rho_{c}=\sigma_{v}^{2} /\left(\sigma_{v}^{2}+\sigma_{\varepsilon}^{2}\right)=\sigma_{v}^{2} / \sigma_{y_{c}}^{2}$ for the control arm. The correlation between two randomly drawn persons from two different clusters, possibly from two different treatment arms, is zero.

\section{Optimal number of clusters for the treatment effect under a cost constraint}

Let $\xi=\left(m, n, K_{t}, K_{c}\right)$ denote the design of a randomized trial with clustering in both arms, and let $\operatorname{var}\left(\hat{\beta}_{1} \mid \xi\right)$ be the asymptotic variance of the estimator of treatment effect $\beta_{1}$, given this design $\xi$. As can be derived from equation (14) in Appendix A, the asymptotic variance of the maximum likelihood estimator (and of the restricted maximum likelihood (REML) estimator, see Demidenko ${ }^{12}$ ) of the treatment effect, $\hat{\beta}_{1}$, is given by

$$
\operatorname{var}\left(\hat{\beta}_{1} \mid \xi\right)=\left((m-1) \rho_{t}+1\right)\left(\frac{\sigma_{y_{t}}^{2}}{m K_{t}}\right)+\left((n-1) \rho_{c}+1\right)\left(\frac{\sigma_{y_{c}}^{2}}{n K_{c}}\right)
$$

This variance increases as a function of the intraclass correlations $\rho_{t}$ and $\rho_{c}$ and the variances within the treatment arms $\sigma_{y_{t}}^{2}$ and $\sigma_{y_{c}}^{2}$, and decreases as a function of the cluster sizes $m$ and $n$ and the number of clusters $K_{t}$ and $K_{c}$. Note that the variance of the treatment effect estimator in equation (2) is equal to the sum of the variances of the outcome mean estimator in the treatment condition and of the outcome mean estimator in the control condition. Furthermore, the factors $(m-1) \rho_{t}+1$ and $(n-1) \rho_{c}+1$ are design effects reflecting how much larger these variances are compared to variances for data where there is no clustering.

We derive a design, that is, $\xi=\left(m, n, K_{t}, K_{c}\right)$, that minimizes the variance of the treatment estimator in equation (2) and thus maximizes test power for a fixed budget. We call this the optimal design. Finding optimal designs requires a cost function specifying the relation between the total budget required and the costs per subject/person/patient/individual and per cluster/group in a trial. Let $C_{0}$ be the overhead costs of the trial, that is, all costs which do not depend on the sample size. Let $c_{t}$ and 
$s_{t}$ be the costs per cluster and per subject in the treatment arm respectively, and let $c_{c}$ and $s_{c}$ be the cost per cluster and per subject in the control arm. The total study cost $C$ then equals

$$
C=C_{0}+K_{t}\left(c_{t}+m s_{t}\right)+K_{c}\left(c_{c}+n s_{c}\right)
$$

This cost function can be used as constraint on the total study cost when maximizing the power and precision for the treatment effect, or to minimize the total study cost while keeping power and precision constant. As a special case of the latter aim, if one is interested in minimizing the total number of subjects sampled, that is, $K_{t} m+K_{c} n$, let $s_{t}=s_{c}=1$ and $c_{t}=c_{c}=0$. Further, for individually randomized group treatment trials, there often is an ideal group size, or, for cluster randomized trials, there may be practical limitations leading to a fixed group size for each of the treatments (e.g. a school class with all of its pupils participating in a prevention trial). In such cases, we may consider $m$ and $n$ fixed and can rewrite equation (3) as

$$
C=C_{0}+K_{t} c_{t}^{*}+K_{c} c_{c}^{*}
$$

where $c_{t}^{*}=c_{t}+m s_{t}$ and $c_{c}^{*}=c_{c}+n s_{c}$ are the total costs of a treatment and control cluster. Starting from a fixed budget and fixed cluster/group size for each of the treatment arms, it can be proven (see Appendix A) that the following cluster numbers minimize the variance of the treatment estimator in equation (2)

$$
\begin{aligned}
& K_{t}^{o p t}=\frac{\left(C-C_{0}\right)}{\sqrt{c_{c}^{*} c_{t}^{*}}\left(\sqrt{\left(\frac{\sigma_{y_{c}}^{2}}{\sigma_{y_{t}}^{2}}\right)\left(\frac{m}{n}\right)\left(\frac{(n-1) \rho_{c}+1}{(m-1) \rho_{t}+1}\right)}\right)+c_{t}^{*}}, \text { and } \\
& K_{c}^{o p t}=\frac{\left(C-C_{0}\right)}{\sqrt{c_{c}^{*} c_{t}^{*}}\left(\sqrt{\left(\frac{\sigma_{y_{t}}^{2}}{\sigma_{y_{c}}^{2}}\right)\left(\frac{n}{m}\right)\left(\frac{(m-1) \rho_{t}+1}{(n-1) \rho_{c}+1}\right)}\right)+c_{c}^{*}}
\end{aligned}
$$

from which it follows, after some rewriting, that the ratio of optimal cluster numbers is equal to

$$
\frac{K_{t}^{o p t}}{K_{c}^{o p t}}=\sqrt{\left(\frac{\sigma_{y_{t}}^{2}}{\sigma_{y_{c}}^{2}}\right)\left(\frac{n}{m}\right)\left(\frac{(m-1) \rho_{t}+1}{(n-1) \rho_{c}+1}\right)} \sqrt{\frac{c_{c}^{*}}{c_{t}^{*}}}
$$

This formula extends the allocation ratio derived by Walwyn and Roberts, ${ }^{9}$ which minimizes the variance for a given total sample size (that is, $s_{t}=s_{c}=1$ and $c_{t}=c_{c}=0$ in equation (3)). As can be seen, the number of treatment clusters compared to the number of control clusters increases as a function of the total variance and the intraclass correlation of the treatment condition, and decreases as a function of the cluster size and the cost per cluster in the treatment condition.

For practical purposes, it is useful to transform the optimal numbers of clusters in equation (5) into expressions for numbers of clusters that yield sufficient power for an intervention effect of a particular size. This will be addressed in the next section.

\section{Optimal number of clusters for a given power and effect size}

The test statistic for the effectiveness of the treatment is $\hat{\beta}_{1} / \sqrt{v \hat{a} r\left(\hat{\beta}_{1} \mid \xi\right)}$. In case of REML estimation (without truncation of negative estimates of the variance components), the test statistic 
$\hat{\beta}_{1} / \sqrt{\operatorname{var}\left(\hat{\beta}_{1} \mid \xi\right)}$ can be shown to be identical to a $t$-test for two independent samples on the cluster means (see supplementary materials for a proof). As a result, when the arms are homogeneous in their intraclass correlation, outcome variance, and cluster size, the test statistic follows Student's $t$-distribution with $K_{t}+K_{c}-2$ degrees of freedom. With heterogeneous variances, intraclass correlations or cluster sizes, the cluster means are very likely to have heterogeneous variances, in which case the test statistic can be approximated by a $t$-distribution, the degrees of freedom being calculated according to Satterthwaite's method. ${ }^{13}$ For sufficiently large numbers of clusters (the small $K$ case is covered in section 6), the standard normal is an appropriate reference distribution for this statistic. ${ }^{14}$ The relation between the variance of the treatment estimator and the power level $1-\gamma$ to detect a treatment effect in a two-tailed test with type I error rate $\alpha$, is then approximated by

$$
\operatorname{var}\left(\hat{\beta}_{1} \mid \xi\right)=\left(\frac{\beta_{1}}{z_{1-\alpha / 2}+z_{1-\gamma}}\right)^{2}
$$

where $z_{1-\alpha / 2}$ and $z_{1-\gamma}$ are the $100(1-\alpha / 2)$ and $100(1-\gamma)$ percentiles of the standard normal distribution. Let $\psi$ be the ratio of the variance in the treatment arm versus the variance in the control arm, $\psi=\sigma_{y_{t}}^{2} / \sigma_{y_{c}}^{2}$. Furthermore, let $E S=\beta_{1} / \sqrt{0.5\left(\sigma_{y_{t}}^{2}+\sigma_{y_{c}}^{2}\right)}$ be the effect size based on the variances in the treatment and control arm (cf. Cohen ${ }^{15}$ ). From equations (2) and (7), the optimal number of clusters for the treatment arm that realizes a certain power level $1-\gamma$ can then be derived (see Appendix A)

$$
K_{t}^{o p t}=\frac{1}{E S^{2}}\left(z_{1-\alpha / 2}+z_{1-\gamma}\right)^{2} \sqrt{\frac{(m-1) \rho_{t}+1}{m}}\left(\sqrt{\frac{(m-1) \rho_{t}+1}{m}}+\sqrt{\frac{(n-1) \rho_{c}+1}{\psi n} \times \frac{c_{c}^{*}}{c_{t}^{*}}}\right)\left(\frac{2 \psi}{\psi+1}\right)
$$

Substituting this expression into equation (6), yields the optimal number of clusters in the control arm, $K_{c}^{\text {opt }}$, for a power level of $1-\gamma$

$$
K_{c}^{o p t}=\frac{1}{E S^{2}}\left(z_{1-\alpha / 2}+z_{1-\gamma}\right)^{2} \sqrt{\frac{(n-1) \rho_{c}+1}{n}}\left(\sqrt{\frac{(n-1) \rho_{c}+1}{n}}+\sqrt{\frac{(m-1) \rho_{t}+1}{m} \times \psi \times \frac{c_{t}^{*}}{c_{c}^{*}}}\right)\left(\frac{2}{\psi+1}\right)
$$

Equations (8) and (9) show that both $K_{t}^{o p t}$ and $K_{c}^{o p t}$ increase with $\rho_{t}$ and $\rho_{c}$, whereas $K_{t}^{o p t}$ decreases and $K_{c}^{o p t}$ increases with $c_{t}^{*} / c_{c}^{*}$. It should be noted that these effects of the intraclass correlation hold for a given power and $E S$, since increasing either of the intraclass correlations increases the variance of the treatment effect estimator (see equation (2)). Under a budget constraint on the other hand, increasing one intraclass correlation increases the number of clusters for the corresponding arm at the expense of the number of clusters of the other arm, see equation (6). How the optimal numbers of clusters change when $m$ and $n$ change, with $c_{t}^{*} / c_{c}^{*}$ in equations (8) and (9) changing correspondingly, is somewhat more complicated. Taking the derivative of equation (8) with respect to $m$ and $n$ shows that $K_{t}^{o p t}$ always decreases as a function of $m$, but, as a function of $n$, is minimum at $n=\left(\left(c_{c} / s_{c}\right)\left(1-\rho_{c}\right) / \rho_{c}\right)^{1 / 2}$. In a similar way, it can be shown that $K_{c}^{\text {opt }}$ always decreases as a function of $n$, but, as a function of $m$, takes a minimum at $m=\left(\left(c_{t} / s_{t}\right)\left(1-\rho_{t}\right) / \rho_{t}\right)^{1 / 2}$.

For a fixed treatment effect, $\beta_{1}$, and a fixed outcome variance summed across both arms, that is, $\sigma_{y_{t}}^{2}+\sigma_{y_{c}}^{2}$, it can further be shown that $K_{t}^{o p t}$ and $K_{c}^{o p t}$ have a maximum as a function of $\psi$, with the 
maximum for $K_{t}^{\text {opt }}$ occurring for values $\psi \geq 1$ and the maximum for $K_{c}^{\text {opt }}$ occurring for values $\psi \leq 1$. More precisely, the maximizer for $K_{t}^{o p t}$ as a function of $\psi$ is

$$
\psi_{\max }=\left(\frac{(m-1) \rho_{t}+1}{(n-1) \rho_{c}+1}\right) \times \frac{n}{m} \times \frac{c_{t}^{*}}{c_{c}^{*}} \times\left(\sqrt{1+\left(\frac{(n-1) \rho_{c}+1}{(m-1) \rho_{t}+1}\right) \times \frac{m}{n} \times \frac{c_{c}^{*}}{c_{t}^{*}}}+1\right)^{2}
$$

and the maximizer for $K_{c}^{o p t}$ is

$$
\psi_{\max }=\left(\frac{(n-1) \rho_{c}+1}{(m-1) \rho_{t}+1}\right) \times \frac{m}{n} \times \frac{c_{c}^{*}}{c_{t}^{*}} \times\left(\sqrt{1+\left(\frac{(m-1) \rho_{t}+1}{(n-1) \rho_{c}+1}\right) \times \frac{n}{m} \times \frac{c_{t}^{*}}{c_{c}^{*}}}-1\right)^{2}
$$

Figure 1 illustrates the effect of increasing $\psi$, for a scenario where $E S=0.5$ (i.e. a medium sized effect according to Cohen ${ }^{15}$ ). For the range of values shown for $\psi$, increasing $\psi$ leads to a decrease of $K_{c}^{o p t}$, but first leads to an increase and then to a decrease of $K_{t}^{o p t}$. Again these trends apply under the constraint of a fixed $E S$ and power. When fixing the budget, increasing $\psi$ always leads to an increase of $K_{t}$ and a decrease of $K_{c}$ (see equation (6)). Furthermore, in line with equations (8) and (9), for both arms the optimal number of clusters increases as $\rho_{c}$ increases from 0.01 to 0.30 ( $\rho_{t}$ in both cases being fixed at 0.01 in Figure 1).

Table 1 presents, for several cost ratios, examples of the optimal numbers of clusters for various values of the variance ratios, cluster sizes $m$ and $n$, and intraclass correlations (i.e. $\rho_{t}=\rho_{c}=0.01$ and $\left.\rho_{t}=\rho_{c}=0.30\right)$. Following Raudenbush ${ }^{1}$ and Van Breukelen and Candel, ${ }^{16}$ we consider the cost ratios $c_{t} / s_{t}$ and $c_{c} / s_{c}$ to vary between 2 and 50, and, in line with Liu, ${ }^{11} s_{t} / s_{c}$ varies between $1 / 10$ and 10. Employing the standard normal distribution as the reference distribution for the test

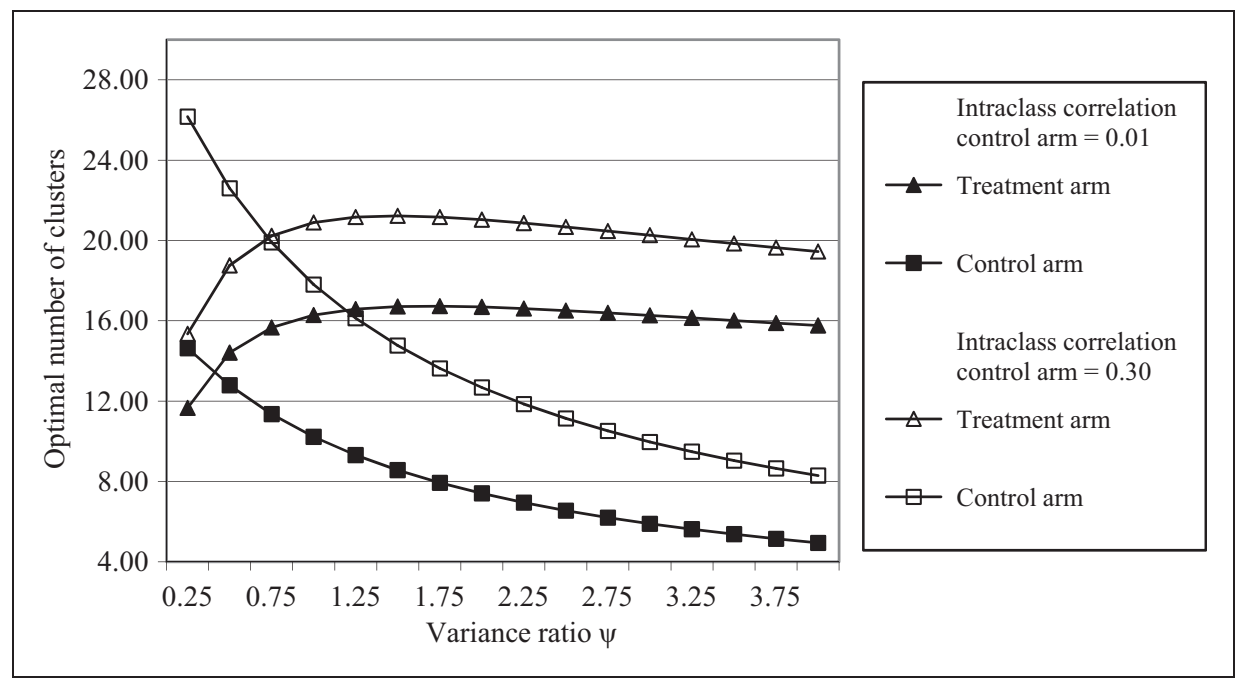

Figure I. Optimal number of clusters for the treatment and control arm for $m=10, n=4, \rho_{t}=0.0 \mathrm{I}, c_{t} / s_{t}=c_{c} / s_{c}=5$, $s_{t} s_{c}=0.1$, effect size $(E S)=0.5$, power $=0.80$, and type I error rate $=0.05$, as a function of the variance ratio $\psi$ and intraclass correlation in the control arm $\left(\rho_{c}=0.01\right.$ versus $\left.\rho_{c}=0.30\right)$. 
Table I. Optimal cluster numbers for different cost ratios, variance ratios, cluster sizes $m$ and $n$, and different intraclass correlations, for an effect size $E S=0.50$, assuming a power of $80 \%$ and a two-tailed test with type I error rate $\alpha=0.05$.

\begin{tabular}{|c|c|c|c|c|c|c|c|c|c|c|}
\hline \multirow[b]{2}{*}{$c_{t} / s_{t}$} & \multirow[b]{2}{*}{$c_{c} / s_{c}$} & \multirow[b]{2}{*}{$s_{t} / s_{c}$} & \multirow[b]{2}{*}{$c_{t} / c_{c}$} & \multirow[b]{2}{*}{$\psi$} & \multirow[b]{2}{*}{$m$} & \multirow[b]{2}{*}{$n$} & \multicolumn{2}{|c|}{$\rho_{t}=0.01 ; \rho_{c}=0.01$} & \multicolumn{2}{|c|}{$\rho_{t}=0.30 ; \rho_{c}=0.30$} \\
\hline & & & & & & & $K_{t}^{o p t}$ & $K_{c}^{o p t}$ & $K_{t}^{o p t}$ & $K_{c}^{o p t}$ \\
\hline 2 & 2 & 0.1 & 0.1 & 0.25 & 4 & 4 & 24 & 15 & 44 & 28 \\
\hline 2 & 2 & 0.1 & 0.1 & 0.25 & 4 & 16 & 22 & 5 & 62 & 20 \\
\hline 2 & 2 & 0.1 & 0.1 & 0.25 & 16 & 4 & 8 & 15 & 23 & 30 \\
\hline 2 & 2 & 0.1 & 0.1 & 0.25 & 16 & 16 & 7 & 5 & 34 & 20 \\
\hline 2 & 2 & 0.1 & 0.1 & 4 & 4 & 4 & 34 & 6 & 62 & 10 \\
\hline 2 & 2 & 0.1 & 0.1 & 4 & 4 & 16 & 32 & 2 & 80 & 7 \\
\hline 2 & 2 & 0.1 & 0.1 & 4 & 16 & 4 & 10 & 6 & 36 & 12 \\
\hline 2 & 2 & 0.1 & 0.1 & 4 & 16 & 16 & 10 & 2 & 45 & 8 \\
\hline 50 & 2 & 0.1 & 2.5 & 0.25 & 4 & 4 & 11 & 20 & 19 & 36 \\
\hline 50 & 2 & 0.1 & 2.5 & 0.25 & 16 & 4 & 5 & 17 & 14 & 35 \\
\hline 50 & 2 & 0.1 & 2.5 & 0.25 & 4 & 16 & 10 & 6 & 25 & 23 \\
\hline 50 & 2 & 0.1 & 2.5 & 0.25 & 16 & 16 & 4 & 5 & 19 & 23 \\
\hline 50 & 2 & 0.1 & 2.5 & 4 & 4 & 4 & 20 & 10 & 37 & 18 \\
\hline 50 & 2 & 0.1 & 2.5 & 4 & 16 & 4 & 7 & 7 & 27 & 17 \\
\hline 50 & 2 & 0.1 & 2.5 & 4 & 4 & 16 & 20 & 3 & 43 & 10 \\
\hline 50 & 2 & 0.1 & 2.5 & 4 & 16 & 16 & 7 & 2 & 32 & 10 \\
\hline 50 & 2 & 2 & 50 & 0.25 & 4 & 4 & 5 & 41 & 9 & 75 \\
\hline 50 & 2 & 2 & 50 & 0.25 & 16 & 4 & 2 & 29 & 7 & 72 \\
\hline 50 & 2 & 2 & 50 & 0.25 & 4 & 16 & 5 & 12 & 11 & 43 \\
\hline 50 & 2 & 2 & 50 & 0.25 & 16 & 16 & 2 & 9 & 8 & 41 \\
\hline 50 & 2 & 2 & 50 & 4 & 4 & 4 & 15 & 31 & 27 & 57 \\
\hline 50 & 2 & 2 & 50 & 4 & 16 & 4 & 5 & 20 & 20 & 54 \\
\hline 50 & 2 & 2 & 50 & 4 & 4 & 16 & 15 & 10 & 28 & 30 \\
\hline 50 & 2 & 2 & 50 & 4 & 16 & 16 & 5 & 6 & 21 & 28 \\
\hline 50 & 50 & 0.1 & 0.1 & 0.25 & 4 & 4 & 24 & 15 & 44 & 28 \\
\hline 50 & 50 & 0.1 & 0.1 & 0.25 & 16 & 4 & 11 & 15 & 34 & 28 \\
\hline 50 & 50 & 0.1 & 0.1 & 0.25 & 4 & 16 & 16 & 5 & 42 & 21 \\
\hline 50 & 50 & 0.1 & 0.1 & 0.25 & 16 & 16 & 7 & 5 & 32 & 20 \\
\hline 50 & 50 & 0.1 & 0.1 & 4 & 4 & 4 & 34 & 6 & 62 & 10 \\
\hline 50 & 50 & 0.1 & 0.1 & 4 & 16 & 4 & 14 & 5 & 47 & 10 \\
\hline 50 & 50 & 0.1 & 0.1 & 4 & 4 & 16 & 25 & 2 & 60 & 8 \\
\hline 50 & 50 & 0.1 & 0.1 & 4 & 16 & 16 & 10 & 2 & 45 & 8 \\
\hline
\end{tabular}

statistic, optimal cluster numbers are given for $80 \%$ power for a medium $E S$, that is, $E S=0.5$. As can be seen, the variation in optimal cluster numbers is rather large and depends on the different parameters in the way as delineated above. For example, the optimal numbers of clusters are always larger for larger intraclass correlations. Also, increasing $m$ and $n$, respectively, leads to decrease in $K_{t}^{\text {opt }}$ and $K_{c}^{\text {opt }}$. It can also be seen that the optimal allocation ratio of treatment and control clusters may clearly deviate from one, contrasting with common practice..$^{7,17-19}$

Table 1 furthermore illustrates that the optimal number of clusters for one of the arms may become larger than feasible. In these cases, the number of clusters in this arm could be reduced to the largest number that is feasible, and the number of clusters in the other arm then needs to be 
increased such that the power level is maintained according to equations (2) and (7). This would then be the most cost-efficient feasible design.

Calculation of optimal designs yielding sufficient power requires knowledge of relevant model parameters such as the intraclass correlations $\rho_{t}$ and $\rho_{c}$ and the variance ratio $\psi$. Since there will rarely be precise knowledge on these parameters, in the next section, we will discuss the maximin design as a possible solution.

\section{Uncertainty on model parameters: Maximin designs}

To calculate the optimal cluster numbers by equations (8) and (9), we need to specify the values of the intraclass correlations $\rho_{t}$ and $\rho_{c}$ and the value of $\psi$, on which we may have no precise prior knowledge. A rather straightforward solution is the maximin strategy. ${ }^{20}$ The maximin strategy consists of the following two steps: (1) for each design determine the minimum efficiency of the treatment effect estimator across the range of plausible values for the intraclass correlations and variance ratio $\psi$, and (2) choose that design, which, given the budget, maximizes this minimum efficiency. This design optimizes a worst case scenario and is known as the maximin design. Since efficiency is the inverse of the variance of the treatment estimator, the maximin strategy implies choosing that design which minimizes the maximum variance of the treatment effect estimator. This is practically useful because, in sample size calculation, choosing values for the intraclass correlations and the variance ratio which maximize the variance of the treatment estimator, will guarantee a desired power level also for all other values of these parameters within their plausible ranges. The maximin design is obtained by filling in these worst case intraclass correlations and variance ratio in equations (8) and (9), and thereby guarantees the power level under the worst case scenario at the lowest costs.

Taking the derivative of equation (2) with respect to $\rho_{t}$ and $\rho_{c}$ shows that the variance of the treatment effect estimator increases as a function of both $\rho_{t}$ and $\rho_{c}$. The maximin design therefore should be based on the upper boundaries of the plausible ranges for these parameters. Keeping the total outcome variance, that is $\sigma_{y_{t}}^{2}+\sigma_{y_{c}}^{2}$, constant, we furthermore show in Appendix B that the variance of the treatment estimator in the maximin design is maximized by the following value of $\psi$

$$
\psi_{\max }=\left(\frac{(m-1) \rho_{t}+1}{m}\right)\left(\frac{n}{(n-1) \rho_{c}+1}\right) \frac{c_{t}^{*}}{c_{c}^{*}}
$$

If the range of plausible values for $\psi$ comprises equation (12), then this value should be chosen, if the range is below the value in equation (12), then the upper boundary of the range should be chosen, and if the range is above the value in equation (12), then the lower boundary of the range should be chosen. These choices give the maximum variance of the treatment estimator in equation (2), and therefore yield safe sample sizes by equations (8) and (9).

Note that equations (8) and (9) are based on the standard normal as a reference distribution for the test statistic. Hence, when small numbers of clusters result, corrections may be needed of these cluster numbers to obtain the desired power level. This issue will be addressed in the next section.

\section{Corrections for the numbers of clusters as based on the normal approximation}

The sample size calculations in equations (8) and (9) are based on the standard normal approximation of the test statistic. In case the treatment arms have equal outcome variances, equal intracluster correlations and equal cluster sizes, so that the variance of cluster outcome 
means is homogeneous between arms, numerical evaluations for $80 \%$ and $90 \%$ power show that, for $K_{t}=K_{c}$ varying from five to 50 , adding one cluster to $K_{t}$ and $K_{c}$ each, is a sufficient correction for two-tailed tests with a 5\% type I error rate. Adding two clusters to $K_{t}$ and $K_{c}$ each turns out to be a sufficient correction for a $1 \%$ type I error rate. ${ }^{16}$

In case the outcome variances, intracluster correlations or cluster sizes are not the same across both treatment arms, an adequate approximation of the distribution of the test statistic, provided each treatment arm contains at least four clusters, ${ }^{13,21}$ is the $t$-distribution with degrees of freedom given by the Satterthwaite approximation. Employing an exact expression for the power of this Satterthwaite approximation, ${ }^{22}$ a numerical evaluation for heterogeneous treatment arms was done to determine appropriate corrections of the number of clusters. Since the numerical evaluation has to be based on realistic ranges for each of the parameters in equations (8) and (9), these parameter ranges will be addressed first.

\section{I Choice of ranges for relevant model parameters}

\section{I.I Intraclass correlations}

The intraclass correlations, $\rho_{t}$ and $\rho_{c}$, range from 0.01 to 0.30 , since this represents the range commonly encountered in trials comparing group administered interventions ${ }^{2,3,8}$ or trials where clustering effects are due to therapists. 3,23

\subsubsection{Ratio of outcome variances}

Since there is not much empirical evidence on $\psi$, with one study indicating that it varies between 0.5 and $2,{ }^{7}$ we will examine a somewhat broader range: $\psi$ runs from 0.25 to 4 .

\subsubsection{Cluster sizes}

In individually randomized group treatments as well as in case of individual treatments with clustering due to shared therapist effects, the cluster sizes are often rather small. The smallest average group size encountered in group therapy is about four, ${ }^{8,19,24}$ whereas 10 seems to be an upper limit. ${ }^{7,18,25}$ These cluster sizes also encompass the average numbers of patients commonly treated within the same period by one therapist, ${ }^{23,26,27}$ although the maximum seems to be somewhat larger. ${ }^{28,29}$ A cluster size of 16 will therefore be considered as an upper limit.

\subsubsection{Effect sizes}

The ES varies between 0.1 and 0.9, thus encompassing the ESs commonly classified as small (i.e. 0.2 ), medium (i.e. 0.5), and large (i.e. 0.80). ${ }^{15}$

\section{I.5 Cost ratios}

The empirical evidence on the costs $c_{t}, c_{c}, s_{t}$, and $s_{c}$ is rather scarce, an exception being for instance Moerbeek et al. ${ }^{30}$ (where $c_{t} / s_{t}=c_{c} / s_{c}=26$ ). To obtain general results, we therefore did not constrain the number of clusters through the cost function in equation (4). Instead $K_{t}$ and $K_{c}$ were varied from two to 140 , such that they yielded $80 \%$ or $90 \%$ power in a two-tailed test with a $5 \%$ or $1 \%$ type I error rate.

\subsection{Numerical investigation}

First, all different combinations of $\rho_{t}, \rho_{c}, \psi, m, n, E S$, and $K_{c}$ were generated within the ranges of these parameters as described in section 6.1. To obtain $K_{t}$ that, given the values of the other 
parameters, yields a power level $1-\gamma$ in a two-tailed test with type I error rate $\alpha$, equations (2) and (7) are combined to obtain the following expression for $K_{t}$

$$
K_{t}=\frac{\left(z_{1-\alpha / 2}+z_{1-\gamma}\right)^{2}\left(\frac{(m-1) \rho_{t}+1}{m}\right) \psi K_{c}}{0.5 \times E S^{2}(\psi+1) K_{c}-\left(z_{1-\alpha / 2}+z_{1-\gamma}\right)^{2}\left(\frac{(n-1) \rho_{c}+1}{n}\right)}
$$

Second, it was checked whether adding a certain number of clusters to the treatment arms is sufficient for yielding a required power level, as evaluated by an exact expression for the power. ${ }^{22}$ For this check a program in $R$ was written. ${ }^{31}$ For some values of the parameters, the integral function in $R$ did not yield a finite value, in which case 200.000 Monte Carlo simulations were done to evaluate the power level.

A simple correction was determined in which an equal number of clusters was added to both treatment arms. More precisely, the smallest number of clusters was determined that, when added to both treatment arms, was sufficient for all possible combinations of $\rho_{t}, \rho_{c}, \psi, m, n, E S, K_{c}$, and $K_{t}$. Let us denote this smallest number by $k$. Next, refinements of this correction were determined. As a first step, the smallest $K_{t}$ and smallest $K_{c}$ were sought for, for which the (same) number of clusters added to each arm could be reduced to $k-1$. Denote this smallest $K_{t}$ and $K_{c}$ by $K_{l}$. The result of this process is visualized in Figure 2. To find $K_{1}$, a binary search algorithm was used. ${ }^{32}$ If $K_{1}$ had been found, then a second binary search was done to find the smallest $K_{t}$ and smallest $K_{c}$ for which the number of clusters added could be reduced to $k-2$ for both arms. Denote this smallest $K_{t}$ and $K_{c}$ by $K_{2}$ (where $K_{2}>K_{1}$ ). These binary searches where repeated until either a smallest $K_{t}$ and smallest $K_{c}$ was found for which no correction to equation (13) was necessary anymore, or no correction turned out to be sufficient for the required power level even for a $K_{t}$ and $K_{c}$ of 140 .

In a second step, these corrections were further refined as a function of the maximum of $K_{t}$ and $K_{c}$. More precisely, consider the area in Figure 2 for which adding $k-1$ clusters (but not $k-2$ clusters) to each arm was sufficient, that is, $K_{t}$ in $\left[K_{1}, K_{2}-1\right]$ and $K_{c}$ in $\left[K_{1}, 140\right]$ or $K_{c}$ in $\left[K_{1}, K_{2}-1\right]$ and $K_{t}$ in $\left[K_{1}, 140\right]$. For this area, it was examined through a binary search, at which smallest value of the largest of $K_{t}$ and $K_{c}$, the number of clusters added to the largest of $K_{t}$ and $K_{c}$ could be one less (that is, $k-2$ instead of $k-1$ ). If this smallest value was found, say $K_{3}$, next for the area where $K_{t}$ in $\left[K_{1}, K_{2}-1\right]$ but $K_{c}$ in $\left[K_{3}, 140\right]$ or $K_{c}$ in $\left[K_{1}, K_{2}-1\right]$ but $K_{t}$ in $\left[K_{3}, 140\right]$ (see Figure 2), a binary search was done to determine the smallest maximum of $K_{t}$ and $K_{c}$ for which the number of clusters added to the largest arm could be further reduced by one (that is, $k-3$ ). This refinement was carried out for each correction area as established before (the areas defined by $K_{1}$ and $K_{2}$ in Figure 2).

\subsection{Results from the numerical evaluation}

For $K_{t}$ and $K_{c}$ varying from two to 140 , it appears that adding three clusters to $K_{t}$ and $K_{c}$ each, is a sufficient correction in case of two-tailed test at a 5\% type I error rate. For a two-tailed test at a $1 \%$ type I error rate, adding four clusters to $K_{t}$ and $K_{c}$ each turns out to be sufficient. These corrections can be relaxed somewhat in case of larger cluster numbers. Table 2(a) and (b) shows sufficient corrections of the cluster numbers as a function of the minimum of $K_{t}$ and $K_{c}$ on one hand and the maximum of $K_{t}$ and $K_{c}$ on the other. These refinements in corrections guarantee a power level up to $0.5 \%$, that is, at least $79.5 \%$ and $89.5 \%$ for the power levels $80 \%$ and $90 \%$, respectively. As an example, for $80 \%$ power and a $5 \%$ type I error rate adding two clusters to each arm are sufficient corrections when the minimum of $K_{t}$ and $K_{c}$ according to equations (8) and (9) exceeds seven (see Table 2(a)). Furthermore, if one of $K_{t}$ and $K_{c}$ exceeds 68, then for this arm it suffices to add only one 


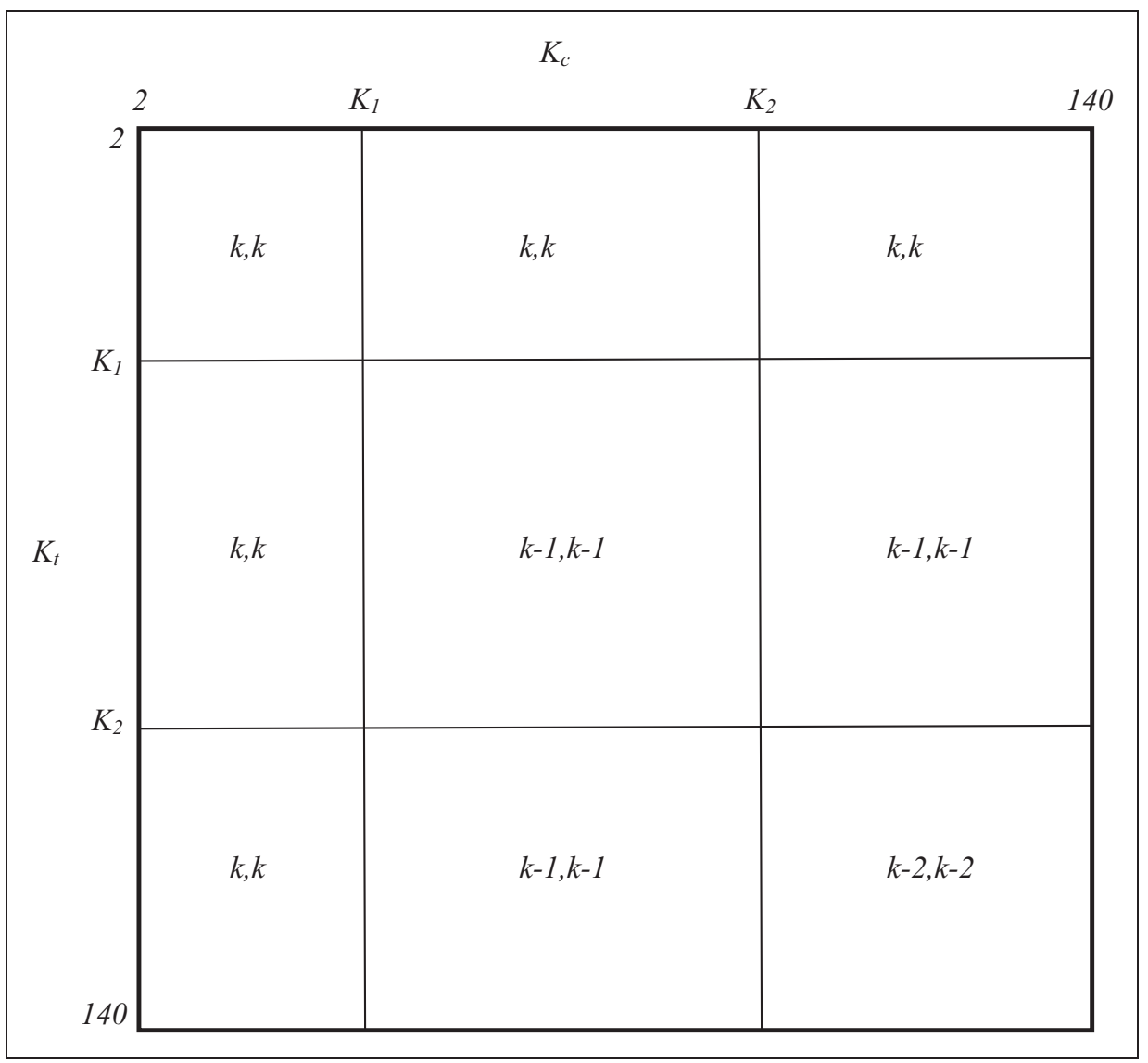

Figure 2. Graphical display of sufficient corrections in terms of adding the same number of clusters to $K_{t}$ and $K_{c}$ as a function of the minimum of $K_{t}$ and $K_{c}$. The ranges for $K_{t}$ and $K_{c}$ run from two to 140. If both $K_{t}$ and $K_{c}$ are $K_{1}$ or larger at most $k-I$ clusters have to be added to $K_{t}$ and to $K_{c}(k-I, k-I)$. If both $K_{t}$ and $K_{c}$ are $K_{2}$ or larger at most $k-2$ clusters have to be added to $K_{t}$ and to $K_{c}(k-2, k-2)$.

cluster. For the same power level but a 1\% type I error rate, it suffices to add three clusters to each arm if the minimum of $K_{t}$ and $K_{c}$ exceeds 25 (see Table 2(b)). If also one of $K_{t}$ and $K_{c}$ exceeds 89, then for this arm only two clusters need to be added.

Below we will illustrate how optimal and maximin sample sizes can be calculated for a specific study, and will also illustrate the benefits of using a maximin design as compared to a design with equal numbers of clusters in each arm.

\section{Application in planning a trial}

We illustrate how to use the results of the present study when planning a trial with heterogeneous clustering in the treatment arms. Baldwin et al. ${ }^{7}$ studied the effects of two group-administered interventions (dissonance intervention versus a healthy weight management program), administered to high school and university students expressing body image concerns. In this study, a central outcome was the score on the Revised Ideal Body Stereotype Scale, a self-report 
Table 2(a). The extra number of clusters to be added to each treatment arm (to the minimum and to the maximum number of clusters), both printed bold within parentheses, when calculating the cluster numbers according to the standard normal approximation in equations (8) and (9) and when employing REML estimation in the linear mixed model analysis.

\begin{tabular}{|c|c|c|c|c|c|}
\hline \multicolumn{2}{|l|}{ Power $=0.80$} & & \multicolumn{2}{|l|}{ Power $=0.90$} & \\
\hline $\begin{array}{l}\text { Minimum of } \\
K_{t} \text { and } K_{c}\end{array}$ & $\begin{array}{l}\text { Maximum of } \\
K_{t} \text { and } K_{c}\end{array}$ & & $\begin{array}{l}\text { Minimum of } \\
K_{t} \text { and } K_{c}\end{array}$ & $\begin{array}{l}\text { Maximum of } \\
K_{t} \text { and } K_{c}\end{array}$ & \\
\hline $2-4$ & $2-4$ & $(+3,+3)$ & $2-3$ & $2-3$ & $(+3,+3)$ \\
\hline $2-7$ & $5-18$ & $(+3,+2)$ & $2-6$ & $4-17$ & $(+3,+2)$ \\
\hline $2-7$ & $19-28$ & $(+3,+1)$ & $2-6$ & $18-26$ & $(+3,+1)$ \\
\hline $2-7$ & $29-140$ & $(+3,+0)$ & $2-6$ & $27-140$ & $(+3,+0)$ \\
\hline $8-68$ & 8-68 & $(+2,+2)$ & $7-53$ & $7-140$ & $(+2,+2)$ \\
\hline $8-74$ & $69-138$ & $(+2,+1)$ & 54-104 & $54-119$ & $(+\vec{I},+I)$ \\
\hline $8-74$ & |39-140 & $(+2,+0)$ & 54-104 & $120-140$ & $(+1,+0)$ \\
\hline $75-140$ & $75-140$ & $(+1,+1)$ & $105-140$ & $105-140$ & $(+0,+0)$ \\
\hline
\end{tabular}

Table 2(b). The extra number of clusters to be added to each treatment arm (to the minimum and to the maximum number of clusters), both printed bold within parentheses, when calculating the cluster numbers according to the standard normal approximation in equations (8) and (9) and when employing REML estimation in the linear mixed model analysis.

\begin{tabular}{|c|c|c|c|c|c|}
\hline \multicolumn{2}{|l|}{ Power $=0.80$} & & \multicolumn{2}{|l|}{ Power $=0.90$} & \\
\hline $\begin{array}{l}\text { Minimum of } \\
K_{t} \text { and } K_{c}\end{array}$ & $\begin{array}{l}\text { Maximum of } \\
K_{t} \text { and } K_{c}\end{array}$ & & $\begin{array}{l}\text { Minimum of } \\
K_{t} \text { and } K_{c}\end{array}$ & $\begin{array}{l}\text { Maximum of } \\
K_{t} \text { and } K_{c}\end{array}$ & \\
\hline $2-17$ & $2-17$ & $(+4,+4)$ & $2-14$ & $2-14$ & $(+4,+4)$ \\
\hline $2-25$ & $18-47$ & $(+4,+3)$ & $2-21$ & $15-35$ & $(+4,+3)$ \\
\hline $2-25$ & $48-64$ & $(+4,+2)$ & $2-21$ & $36-57$ & $(+4,+2)$ \\
\hline $2-25$ & $65-93$ & $(+4,+1)$ & $2-21$ & $58-8 \mid$ & $(+4,+1)$ \\
\hline $2-25$ & $94-140$ & $(+4,+0)$ & $2-21$ & $82-140$ & $(+4,+0)$ \\
\hline $26-89$ & $26-89$ & $(+3,+3)$ & $22-70$ & $22-70$ & $(+3,+3)$ \\
\hline $26-94$ & $90-139$ & $(+3,+2)$ & $22-73$ & $7|-| 3 \mid$ & $(+3,+2)$ \\
\hline $26-94$ & $|40-| 40$ & $(+3,+1)$ & $22-73$ & $132-140$ & $(+3,+1)$ \\
\hline \multirow[t]{3}{*}{$95-140$} & $95-140$ & $(+2,+2)$ & $74-132$ & $74-139$ & $(+2,+2)$ \\
\hline & & & $74-132$ & $140-140$ & $(+2,+1)$ \\
\hline & & & $133-140$ & $133-140$ & $(+I,+I)$ \\
\hline
\end{tabular}

measure of internalization of the thin beauty ideal. Equations (8) and (9) can be used to calculate the numbers of clusters when replicating this study. We set $s_{t}=s_{c}=1$ and $c_{t}=c_{c}=0$ in equation (3) so that the total sample size is minimized and we take the parameter estimates from Baldwin et al., ${ }^{7}$ that is, $\hat{\psi}=0.78, \hat{\rho}_{t}=0.04$, and $\hat{\rho}_{c}=0.25$, as best guesses as to the true parameter values. If we want to detect a medium sized effect of the intervention, that is, $E S=0.5\left(\right.$ cf. Cohen $^{15}$ ), with $80 \%$ power at 
Table 3. Budget and power of the maximin design versus an equal allocation design $\left(K_{t}=K_{c}\right)$ for different cost ratios and power levels.

\begin{tabular}{|c|c|c|c|c|c|c|c|}
\hline \multirow[b]{2}{*}{$c_{t} / s_{t}$} & \multirow[b]{2}{*}{$c_{c} / s_{c}$} & \multirow[b]{2}{*}{$s_{t} / s_{c}$} & \multirow[b]{2}{*}{$c_{t} / c_{c}$} & \multicolumn{2}{|c|}{$\begin{array}{l}\text { Relative budget gain } \\
\text { under equal power levels } \\
\text { Power for equal allocation } \\
\text { and maximin design }\end{array}$} & \multicolumn{2}{|c|}{$\begin{array}{l}\text { Absolute power gain } \\
\text { under equal budgets } \\
\text { Power for equal } \\
\text { allocation design }\end{array}$} \\
\hline & & & & $80 \%$ & $90 \%$ & $80 \%$ & $90 \%$ \\
\hline 2 & 2 & 0.1 & 0.1 & $2 \%$ & $3 \%$ & $2 \%$ & $6 \%$ \\
\hline 2 & 2 & I & I & $4 \%$ & $4 \%$ & $2 \%$ & $6 \%$ \\
\hline 2 & 2 & 10 & 10 & $40 \%$ & $41 \%$ & $16 \%$ & $10 \%$ \\
\hline 2 & 20 & 0.1 & 0.01 & $11 \%$ & $10 \%$ & $4 \%$ & $7 \%$ \\
\hline 2 & 20 & I & 0.1 & $-2 \%$ & $-2 \%$ & $-1 \%$ & $5 \%$ \\
\hline 2 & 20 & 10 & I & $19 \%$ & $21 \%$ & $9 \%$ & $8 \%$ \\
\hline 20 & 2 & 0.1 & 1 & $-1 \%$ & $-1 \%$ & $-1 \%$ & $5 \%$ \\
\hline 20 & 2 & 1 & 10 & $20 \%$ & $23 \%$ & $9 \%$ & $9 \%$ \\
\hline 20 & 2 & 10 & 100 & $50 \%$ & $53 \%$ & $19 \%$ & $10 \%$ \\
\hline 20 & 20 & 0.1 & 0.1 & $2 \%$ & $3 \%$ & $2 \%$ & $6 \%$ \\
\hline 20 & 20 & 1 & 1 & $4 \%$ & $4 \%$ & $2 \%$ & $6 \%$ \\
\hline 20 & 20 & 10 & 10 & $40 \%$ & $41 \%$ & $16 \%$ & $10 \%$ \\
\hline
\end{tabular}

The cluster sizes are $m=n=6$, the variance ratio is $\psi=0.6$, the intraclass correlations are $\rho_{t}=0.10$ and $\rho_{c}=0.30$, the effect size is $E S=0.50$ and the type I error rate is $\alpha=0.05$ for a two-tailed test. Shown are the relative budget gains and absolute power gains of the maximin design.

a 5\% type I error rate in a two-tailed test, and $m=n=6$ are ideal group sizes for both arms (cf. Baldwin et al. ${ }^{7}$ ), then $K_{t}=15$ groups are needed for the treatment arm (by equation (8)) and $K_{c}=22$ groups for the other arm (by equation (9)). To repair the power loss due to using the standard normal instead of the $t$-distribution in calculating $K_{t}$ and $K_{c}$, these numbers should be increased to $K_{t}=15+2=17$ and $K_{c}=22+2=24$ groups, respectively (see Table 2(a)).

If one wants to be on the safe side, the maximin strategy can be used, and 0.10 and 0.30 could be chosen as upper boundaries for $\rho_{t}$ and $\rho_{c}$, respectively. Substituting these into equation (12) shows that the variance of the treatment estimator is maximum at $\psi=0.6$. If this value is within the plausible range of values for $\psi$, then, by using equations (8) and (9), the maximin sample sizes can be calculated as: $K_{t}=16$ and $K_{c}=27$. Accounting again for the power loss due to using the standard normal distribution for the test statistic, this should be increased to $K_{t}=16+2=18$ and $K_{c}=27+2=29$ groups, respectively (see Table 2(a)). Calculations of the number of clusters according to an optimal design or a maximin design, also including the corrections as displayed in Table 2(a) and (b), are possible via a small menu-driven program written in $R$ (CLUSCALCRT), ${ }^{31}$ which, upon request, is available from the first author.

Finally, if one wants to replicate the study of Baldwin et al., ${ }^{7}$ one could start from a maximin strategy with $\rho_{t}=0.10, \rho_{c}=0.30$ and $\psi=0.6$, and compare the maximin design to a design where $K_{t}=K_{c}$, as typical for many studies ${ }^{7,17-19}$. Since we do not have information on the research costs, different cost ratios are considered. Columns 5 and 6 of Table 3 show the relative gains in budget when considering a maximin design as compared to a design with $K_{t}=K_{c}$ in case both designs have $80 \%$ or $90 \%$ power at $E S=0.50$ in a two-tailed test with a $5 \%$ type I error rate. The budget gains can become as large as 50\%, illustrating that there may be clear monetary benefits for a maximin design. Further, columns 7 and 8 of Table 3 show the gains in power under identical budgets when 
considering a maximin design as compared to a design with $K_{t}=K_{c}$ which has either $80 \%$ or $90 \%$ power at $E S=0.50$ in a two-tailed test with a $5 \%$ type I error rate. The power gains may be up to nearly $20 \%$ for an $80 \%$ power level and up to $10 \%$ for a $90 \%$ power level of the equal allocation design. This implies that for some cost ratios the power levels for the maximin design are close to $100 \%$ for the same budget as the equal allocation design. Note furthermore that there also may be some small budget and power losses for the maximin design. This is due to rounding of the cluster numbers and due to the corrections as displayed in Table 2(a) and (b) being sufficient but not necessary.

\section{Conclusions and discussion}

Clustering may occur when comparing group administered treatments, such as group wise psychotherapy where group members influence each other, or when comparing individual treatments where individuals are nested within care providers, such as psychotherapists, and provider effects occur. Starting from a priori fixed and constant cluster sizes and employing a flexible cost function, we derived the numbers of clusters that minimize the variance of the treatment effect estimator. This generalizes previous studies in that allowance is made for between-arm differences in the intraclass correlation, outcome variance, cluster size, number of clusters, and costs. The optimal number of clusters for a treatment arm may vary to a large extent (from two to more than 70) and the optimal allocation of clusters to the treatment arms may clearly deviate from a 50-50 allocation. Calculating optimal sample sizes requires prior knowledge on the intraclass correlations in both arms and on the between-arm ratio of outcome variances, which often may not be very precise. To accommodate this, a maximin strategy for calculating sample sizes was presented. Maximin sample sizes guarantee a desired power level at the lowest costs for all parameter values in their plausible ranges. Since the power of any design, including an optimal or a maximin design, also depends on the ES of interest, expressions were furthermore given for calculating the number of clusters needed for a certain power level and $E S$. As these equations were based on the normal approximation of the test statistic, a numerical study was done which showed that for both $80 \%$ and $90 \%$ power, increasing the number of clusters in each arm by either three or four clusters was sufficient to obtain the required power level for type I error rates of $5 \%$ and $1 \%$, respectively.

For some care providers, such as nurse practitioners or general practitioners, the intervention often involves a single or a few meetings of limited duration, implying that the average number of patients per care provider may be much larger than considered in the present numerical study (see e.g. Roberts and Roberts, ${ }^{3}$ Roth et al. ${ }^{33}$ ). Additional numerical evaluations with larger average cluster sizes could be done to examine whether similar corrections result in this case.

The methodology of the present paper could also be applied to designs where the number of clusters are fixed. That is, it is also possible to derive the optimal cluster sizes, for fixed numbers of clusters in the treatment and control arm, by minimizing the variance of the treatment effect estimator for a given budget. However, it may not be possible to find optimal cluster sizes needed for achieving a certain power level, as the variance of the treatment effect estimator does not become arbitrarily small as $m$ and $n$ approach infinity, except if both intraclass correlations are zero (see equation 2). Stated otherwise, given fixed numbers of clusters in the treatment and control arm, there may be no optimal $m$ and $n$ realizing a desired power level for a particular $E S$.

Since the sample size formulas assume constant cluster sizes, whereas varying cluster sizes, due to dropout or naturally varying group sizes, is the more common case, one relevant extension of the paper is examining the effect of varying cluster sizes on the design efficiency and deriving a 
correction for the potential efficiency loss in a cost-efficient way when planning sample sizes. Van Breukelen et al. ${ }^{34}$ have shown that in many cases adding about $10 \%$ of the clusters to both arms compensates the efficiency loss due to varying cluster sizes, but this result applies to arms that are homogeneous in their intraclass correlation, outcome variance, number of clusters and average cluster size. Formulating correction guidelines for the efficiency loss for designs where there is between-arms heterogeneity in these features requires deriving the efficiency loss for the asymptotic case and evaluating the adequacy of this asymptotic result for finite samples through an extensive Monte Carlo simulation study. This extension will be addressed in an upcoming paper.

\section{Funding}

This research received no specific grant from any funding agency in the public, commercial, or not-for-profit sectors.

\section{Conflict of interest}

None declared.

\section{References}

1. Raudenbush SW. Statistical analysis and optimal design for cluster randomized trials. Psychol Meth 1997; 2: 173-185.

2. Baldwin SA, Murray DM and Shadish WR. Empirically supported treatments or type I errors? Problems with the analysis of data from group-administered treatments. J Consult Clin Psychol 2005; 73: 924-935.

3. Roberts $\mathrm{C}$ and Roberts SA. The design and analysis of clinical trials with clustering effects due to treatment. Clin Trials 2005; 2: 152-162.

4. Pals SL, Murray DM, Alfano CM, et al. Individually randomized group treatment trials: a critical appraisal of frequently used design and analytic approaches. $A m J$ Public Health 2008; 98: 1418-1424.

5. Dannon PN, Gon-Usishkin M, Gelbert A, et al. Cognitive behavioral group therapy in panic disorder patients: the efficacy of CBGT versus drug treatment. Ann Clin Psychiatry 2004; 16: 41-46.

6. Otto MW, Pollack MH, Gould RA, et al. A comparison of the efficacy of clonazepam and cognitive-behavioral group therapy for the treatment of social phobia. $J$ Anxiety Disord 2000; 14: 345-358.

7. Baldwin SA, Stice E and Rohde P. Statistical analysis of group-administered intervention data: reanalysis of two randomized trials. Psychother Res 2008; 18: 365-376.

8. Tasca GA, Illing V, Ogrodniczuk JS, et al. Assessing and adjusting for dependent observations in group treatment research using multilevel models. Group Dynam 2009; 13: $151-162$.

9. Walwyn $\mathrm{R}$ and Roberts $\mathrm{C}$. Therapist variation within randomized trials of psychotherapy: implications for precision, internal and external validity. Stat Methods Med Res 2010; 19: 291-315.

10. Moerbeek M, Van Breukelen GJP and Berger MPF. Design issues for experiments in multilevel populations. J Educ Behav Stat 2000; 25: 271-284.

11. Liu X. Statistical power and optimum sample allocation ratio for treatment and control having unequal costs per unit of randomization. $J$ Educ Behav Stat 2003; 28 : 231-248.
12. Demidenko E. Mixed models: theory and applications. New Jersey: Wiley, 2004.

13. Davenport JM and Webster JT. Type-I error and power of a test involving a Satterthwaite's approximate F-statistic. Technometrics 1972; 14: 555-569.

14. Dixon WJ and Massey FM. Introduction to statistical analysis. New York: McGraw Hill, 1983.

15. Cohen J. A power primer. Psychol Bull 1992; 112: 155-159.

16. Van Breukelen GJP and Candel MJJM. Sample sizes for cluster randomized trials: we can keep it simple and efficient! J Clin Epidemiol 2012; 65: 1212-1218.

17. Herzog TA, Lazev AB, Irvin JE, et al. Testing for group membership effects during and after treatment: the example of group therapy for smoking cessation. Behav Ther 2002; 33: 29-43.

18. Hoover DR. Clinical trials of behavioral interventions with heterogeneous teaching subgroup effects. Stat Med 2002; 21: 1351-1364.

19. Tasca GA, Illing V, Joyce AS, et al. Three-level multilevel growth models for nested change data: a guide of group treatment researchers. Psychother Res 2009; 19: 453-461.

20. Atkinson AC, Donev AN and Tobias RD. Optimum experimental designs, with SAS. Oxford: Oxford University Press, 2007.

21. Davenport JM and Webster JT. The Behrens-Fisher problem, an old solution revisited. Metrika 1975; 22: $47-54$.

22. Moser BK, Stevens GR and Watts CL. The two-sample t test versus Satterthwaite's approximate F test. Comm Stat Theor Meth 1989; 18: 3963-3975.

23. De Jong K, Moerbeek M and Van der Leeden R. A priori power analysis in longitudinal three-level multilevel models: an example with therapist effects. Psychother Res 2010; 20: 273-284.

24. Newton-John TRO, Spence SH and Schotte D. Cognitivebehavioral therapy versus EMG feedback in the treatment of chronic low-back-pain. Behav Res Ther 1995; 33 . 691-697.

25. Imel Z, Baldwin $\mathrm{S}$, Bonus $\mathrm{K}$, et al. Beyond the individual: group effects in mindfulness-based stress reduction. Psychother Res 2008; 18: 735-742. 
26. Thompson LW, Gallagher D and Breckenridge JS. Comparative effectiveness of psychotherapies for depressed elders. J Consult Clin Psychol 1987; 55: 385-390.

27. Wampold BE and Serlin RC. The consequence of ignoring a nested factor on measures of effect size in analysis of variance. Psychol Meth 2000; 5: 425-433.

28. Crits-Cristoph P and Mintz J. Implications of therapist effects for the design and analysis of comparative studies of psychotherapies. J Consult Clin Psychol 1991; 59: 20-26.

29. Durham RC, Murphy T, Allan T, et al. Cognitive therapy, analytic psychotherapy and anxiety management training for generalized anxiety disorder. Br J Psychiatry 1994; 165 315-323.

30. Moerbeek M, Van Breukelen GJP, Berger MPF, et al. Optimal sample sizes in experimental designs with individuals nested within clusters. Understand Stat 2003; 2 : 151-175.

31. R Core Team. $R$ : A language and environment for statistical computing. Vienna: R Foundation for Statistical
Computing, http://www.R-project.org/ (2013, accessed 20 November 2014).

32. Cormen TH, Leiserson CE, Rivest RL, et al. Introduction to algorithms. Cambridge, MA: MIT Press, 2009.

33. Roth A, Rogowski O, Yanay Y, et al. Teleconsultation for cardiac patients: a comparison between nurses and physicians. The SHL experience in Israel. Telemed $J E$ Health 2006; 12: 528-534.

34. Van Breukelen GJP, Candel MJJM and Berger MPF. Relative efficiency of unequal versus equal cluster sizes in cluster randomized and multicentre trials. Stat Med 2007; 26: 2589-2603.

35. Candel MJJM and Van Breukelen GJP. Varying cluster sizes in trials with clusters in one treatment arm: sample size adjustments when testing treatment effects with linear mixed models. Stat Med 2009; 28: 2307-2324.

\section{Appendix A: Optimal cluster numbers for fixed cluster sizes in case of ML estimation of the treatment effect}

Let $K$ be the total number of clusters, and let $p$ be the proportion of clusters allocated to the treatment arm (and thus $1-p$ the proportion allocated to the control arm). The asymptotic variance of $\hat{\beta}_{1}$ can, along lines similar to Candel and Van Breukelen, ${ }^{35}$ shown to be

$$
\operatorname{Var}\left(\hat{\beta}_{1}\right)=\frac{1}{K_{t} w_{t}}+\frac{1}{K_{c} w_{c}}
$$

where $w_{t}=\frac{m}{m \sigma_{u}^{2}+\sigma_{\delta}^{2}}$ and $w_{c}=\frac{n}{n \sigma_{v}^{2}+\sigma_{\varepsilon}^{2}}$, which are the inverse variances of a single cluster outcome mean in the treated and control arm respectively. Equation (14) can be rewritten into equation (2) of the main text. From the cost function in equation (4) it follows that $K=\left(C-C_{0}\right) /\left(p c_{t}^{*}+(1-p) c_{c}^{*}\right)$ and equation (14) can then be rewritten as:

$$
\operatorname{Var}\left(\hat{\beta}_{1}\right)=\frac{p K w_{t}+(1-p) K w_{c}}{p K(1-p) K w_{t} w_{c}}=\left(\frac{p w_{t}+(1-p) w_{c}}{p(1-p) w_{t} w_{c}}\right)\left(\frac{p c_{t}^{*}+(1-p) c_{c}^{*}}{\left(C-C_{0}\right)}\right)
$$

Taking the derivative with respect to $p$ and setting the expression to 0 , yields:

$$
\left(\frac{p_{\text {opt }}}{1-p_{\text {opt }}}\right)^{2}=\left(\frac{w_{c}}{w_{t}}\right)\left(\frac{c_{c}^{*}}{c_{t}^{*}}\right) \text {, or }\left(\frac{p_{\text {opt }}}{1-p_{\text {opt }}}\right)=\sqrt{\frac{w_{c}}{w_{t}} \times \frac{c_{c}^{*}}{c_{t}^{*}}}
$$

Since the second derivative of equation (15) is positive, this $p_{\text {opt }}$ minimizes the variance of the treatment effect estimator. Rewriting the cost function in equation (4), noting that $\left(1-p_{\text {opt }}\right) / p_{\text {opt }}=$ $K_{c}^{o p t} / K_{t}^{o p t}$, we obtain:

$$
\begin{aligned}
C-C_{0} & =K_{t}^{o p t} c_{t}^{*}+K_{t}^{o p t}\left(\frac{1-p_{\text {opt }}}{p_{\text {opt }}}\right) c_{c}^{*}, \text { or } \\
K_{t}^{o p t} & =\frac{C-C_{0}}{c_{t}^{*}+\left(\frac{1-p_{\text {opt }}}{p_{\text {opt }}}\right) c_{c}^{*}}=\frac{C-C_{0}}{c_{t}^{*}+\sqrt{c_{t}^{*} c_{c}^{*} \sqrt{\frac{w_{t}}{w_{c}}}} .} .
\end{aligned}
$$


It can be shown that

$$
\frac{w_{t}}{w_{c}}=\left(\frac{\sigma_{y_{c}}^{2}}{\sigma_{y_{t}}^{2}}\right)\left(\frac{m}{n}\right)\left(\frac{(n-1) \rho_{c}+1}{(m-1) \rho_{t}+1}\right),
$$

which, when substituted into equation (17), yields $K_{t}^{\text {opt }}$ in equation (5). A derivation along similar lines can be given for $K_{c}^{\text {opt }}$ in equation (5).

Starting from equations (2) and (7) the expression in equation (8) can now be derived:

$$
\begin{gathered}
\frac{\beta_{1}^{2} K_{t}^{o p t}}{\left(z_{1-\alpha / 2}+z_{1-\gamma}\right)^{2}}=\left\{\frac{\left((m-1) \rho_{t}+1\right) \sigma_{y_{t}}^{2}}{m}+\frac{\left((n-1) \rho_{c}+1\right) \sigma_{y_{c}}^{2}}{n} \times \frac{K_{t}^{o p t}}{K_{c}^{o p t}}\right\}, \text { or } \\
K_{t}^{o p t}=\frac{\left(z_{1-\alpha / 2}+z_{1-\gamma}\right)^{2}}{\beta_{1}^{2}} \times\left\{\frac{\left((m-1) \rho_{t}+1\right) \sigma_{y_{t}}^{2}}{m}+\frac{\left((n-1) \rho_{c}+1\right) \sigma_{y_{c}}^{2}}{n} \times \frac{p_{o p t}}{1-p_{o p t}}\right\},
\end{gathered}
$$

so that, making use of the results in equations (16) and (18)

$$
\begin{aligned}
K_{t}^{o p t}= & \frac{\left(z_{1-\alpha / 2}+z_{1-\gamma}\right)^{2}}{\beta_{1}^{2}} \\
& \times\left\{\frac{\left((m-1) \rho_{t}+1\right) \sigma_{y_{t}}^{2}}{m}+\frac{\left((n-1) \rho_{c}+1\right) \sigma_{y_{c}}^{2}}{n} \sqrt{\left(\frac{n}{m}\right)\left(\frac{c_{c}^{*}}{c_{t}^{*}}\right)\left(\frac{(m-1) \rho_{t}+1}{(n-1) \rho_{c}+1}\right)\left(\frac{\sigma_{y_{t}}^{2}}{\sigma_{y_{c}}^{2}}\right)} .\right.
\end{aligned}
$$

Further elaboration yields:

$$
\begin{aligned}
K_{t}^{o p t}= & \frac{\sigma_{y_{t}}^{2}\left(z_{1-\alpha / 2}+z_{1-\gamma}\right)^{2}}{\beta_{1}^{2}} \sqrt{\frac{\left((m-1) \rho_{t}+1\right)}{m}} \\
& \times\left\{\sqrt{\frac{\left((m-1) \rho_{t}+1\right)}{m}}+\sqrt{\frac{\left((n-1) \rho_{c}+1\right)}{n}\left(\frac{c_{c}^{*}}{c_{t}^{*}}\right)}\left(\frac{\sigma_{y_{c}}}{\sigma_{y_{t}}}\right)\right\},
\end{aligned}
$$
which, after substituting $E S=\beta_{1} / \sqrt{0.5\left(\sigma_{y_{t}}^{2}+\sigma_{y_{c}}^{2}\right)}$ and $\psi=\sigma_{y_{t}}^{2} / \sigma_{y_{c}}^{2}$, yields equation (8) of the
main text.

\section{Appendix B: Value of $\psi$ which maximizes the variance of the treatment effect estimator for maximin designs}

The variance of the treatment effect estimator in equation (2) can be rewritten as:

$$
\operatorname{var}\left(\hat{\beta}_{1} \mid \xi\right)=\left((m-1) \rho_{t}+1\right)\left(\frac{\sigma_{y_{t}}^{2}+\sigma_{y_{c}}^{2}}{m K_{t}}\right)\left(\frac{\psi}{\psi+1}\right)+\left((n-1) \rho_{c}+1\right)\left(\frac{\sigma_{y_{t}}^{2}+\sigma_{y_{c}}^{2}}{n K_{c}}\right)\left(\frac{1}{\psi+1}\right) .
$$

Taking the derivative with respect to $\psi$ shows that $\operatorname{var}\left(\hat{\beta}_{1} \mid \xi\right)$ increases as a function of $\psi$ whenever $\left(\frac{(m-1) \rho_{t}+1}{(n-1) \rho_{c}+1}\right)\left(\frac{n}{m}\right) \geq\left(\frac{K_{t}}{K_{c}}\right)$, and decreases as a function of $\psi$, otherwise. Since the maximin design is an optimal design for a certain value of $\psi, K_{t} / K_{c}$ will satisfy equation (6) and we can derive that $\operatorname{var}\left(\hat{\beta}_{1} \mid \xi\right)$ increases as a function of $\psi$ if $\left(\frac{(m-1) \rho_{t}+1}{(n-1) \rho_{c}+1}\right)\left(\frac{n}{m}\right) \geq \sqrt{\psi\left(\frac{(m-1) \rho_{t}+1}{(n-1) \rho_{c}+1}\right)\left(\frac{n}{m}\right)\left(\frac{c_{c}^{*}}{c_{t}^{*}}\right)}$ and decreases otherwise. The maximum variance of the maximin design is therefore achieved at $\psi=\left(\frac{(m-1) \rho_{t}+1}{(n-1) \rho_{c}+1}\right)\left(\frac{n}{m}\right)\left(\frac{c_{t}^{*}}{c_{c}^{*}}\right)$. 